\title{
Initiatives to Improve Awareness of Delirium in a Teaching Hospital in Singapore: for Better Patient Care
}

\author{
Lim SC*1, Chiam WM ${ }^{2}$, Goh $\mathrm{CH}^{2}$, Zhang $\mathrm{D}^{3}$, Png GK${ }^{3}$ and Li FY ${ }^{3}$ \\ ${ }^{1}$ Department of Geriatric Medicine, singapore \\ ${ }^{2}$ Department of Nursing Informatics, singapore \\ ${ }^{3}$ Department of Nursing, singapore
}

*Corresponding author: Si Ching LIM, Department of Geriatric Medicine, Changi General Hospital, singapore.

Received Date: December 19, 2018

Published Date: January 04, 2019

\begin{abstract}
Delivering good care for the elderly with cognitive issues in a large rapidly turning over acute hospital setting is often challenging at all levels. The elderly patients have multiple medical comorbidities, on multiple medications and often display challenging behaviors which are not easy to manage, unless the care staffs have received training in Gerontology. The onset of challenging behaviors may be the harbinger for something more urgent, and time and care should be promptly instituted, instead of managing the behavior with sedatives or restraints. Diagnosis of delirium is often missed since the symptoms are vague and fluctuate throughout the day. Nurses are perhaps the best partners for the doctors in screening and diagnosing delirium early.
\end{abstract}

Keywords: Elderly; Delirium; Physical restraint; Acute Hospital; Mental state assessment

\section{Introduction}

Singapore is rapidly ageing, the elderly defined as aged 65 and above make up $13 \%$ of the population, and will reach $25 \%$ by 2030 [1]. The elderly account for $1 / 3$ of the total hospital admissions and this figure is on the rising trend. The elderly tend to stay longer in the hospitals once they are admitted and this may contribute to the higher proportion of bed occupancy. New hospitals are being built to increase the bed number, but the acute beds are expensive to run. Cheaper alternatives by expanding the community sectors are being reviewed [2]. The elderly will continue to be the main user of the healthcare resources due to their multiple comorbidities, functional dependency and social issues.

The hospitalized elderly is at risk of developing hospital associated complications such as delirium, falls, under nutrition, immobility and functional decline. These adverse events may start to evolve since the day of admission [3]. Among the complications mentioned, delirium is perhaps the most urgent, since it is potentially fatal and is often under-diagnosed by the medical staff. Collins et al. showed that $72 \%$ of delirium cases were missed by the medical teams within 72 hours of admission to the hospital. While another study by Elie found that $65 \%$ of delirium cases were missed at the emergency department [4,5]. Delirium may be present in up to $50 \%$ of the elderly patients in an acute hospital and carries with it downstream complications such as the risk of dementia, nursing home placement and recurrent episodes of delirium in the subsequent hospital admissions. Patients who developed delirium cost the healthcare system 2.5 times more than their peers who did not develop delirium [6,7].

This is a descriptive paper where the authors were requested to reduce the risk of elderly patients absconding from the hospital, increase delirium and dementia awareness among the staff in the hospital in order to improve the care of the vulnerable elderly and to reduce restraint usage. The authors looked into the point prevalence of the elderly patients $>65$ in the hospital of 1000 beds and estimated the prevalence of confused elderly. The elderly $>65$ occupied $>60 \%$ of the hospital beds and $40-50 \%$ of the elderly patients were estimated to have some cognitive issues of either delirium or dementia. 
The hospital has two main blocks, the larger main building (MB) consists of acute beds for the medical and surgical disciplines and the smaller integrated building (IB) houses the inpatients for the departments of Geriatric Medicine and Rehab medicine. The MB has a mix of young and older patients while IB is made up of mainly elderly patients with a small percentage of young rehab patients $<65$. The proportion of nurses in the MB who are trained in gerontology nursing is much smaller than the nurses in the IB. The nurses are rotated through between MB and IB to gain different experience, but the numbers are not significant and nor is it consistent throughout the years.

\section{Aims}

The aims for this hospital wide project were:

a. Increase awareness of delirium among the nursing and medical staffs.

b. Screen for delirium as soon as there is a change in mental status. c. Early diagnosis and management of delirium.

d. Initiate better care for the confused elderly who are at risk of hospital acquired complications.

e. Avoid usage of physical restraints for the change in behavior.

\section{Clinical Pathway for Delirium}

In order to improve the awareness and early diagnosis of delirium, the project started with creation of a clinical pathway for delirium with screening tools. The tools used were the commonly used Confusion Assessment Method (CAM) and the 4AT [8,9]. Both are validated tools for screening for delirium. However, CAM requires training and is more difficult to use. CAM requires training and the nurses felt that it was subjective and difficult to grasp. The 4AT was simpler and required less training and was generally preferred by the nursing staff. The other components of the delirium clinical pathway include work up for delirium and nursing care protocol for the delirious patients, as shown in Figure 1.
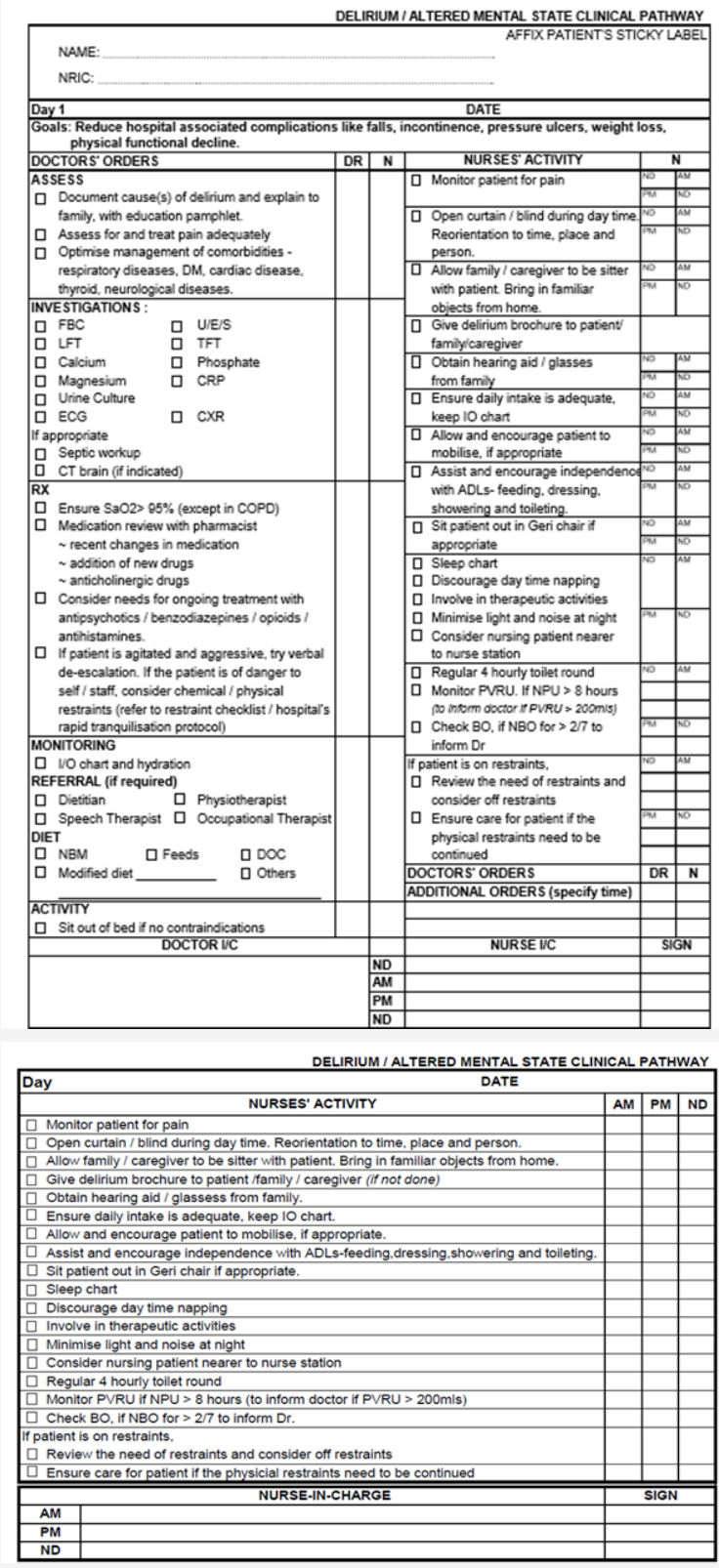

Figure 1: : Delirium pathway. 
CAM and both 4AT were included in the pilot phase to test the nurses' preference. Some of the nurses were familiar with CAM while the others who were not previously exposed were trained for both CAM and 4AT. After the short pilot in various wards in MB and IB, the general consensus was a preference for $4 \mathrm{AT}$. The hard copy pathway was eventually discontinued with a simpler version on the electronic medical records system, see sections below. The hospital is migrating to full electronic record keeping, doing away with paper and documents in hard copy.

\section{Vital Sign Recoding on the Nurses' Flowsheet on Electronic Medical Record System}

The hospital nurses routinely enter the patients' parameters, directly into the hospital's electronic medical system, the SCM.
Within the SCM, the nurses record the patient's heart rate, temperature, oxygen saturation, blood pressure and respiratory rate at least once per shift. The frequency of monitoring and recording may be increased according to doctors' orders when the patients are unwell.

The monitoring of mental status was introduced as the compulsory $6^{\text {th }}$ vital sign, which is cuurently mandatory for all patients at a minimum of once per shift. The mental status assessment is as shown in Figure 2. Once the patient is assessed to be confused or restless, there is a prompt for the patient to be given a purple wrist tag to wear. (Figure 3) The purple wrist tags identify patients who are confused or cognitively impaired. The purple wrist tags (Figure 4) are for easy identification of such patients so the nurses and other hospital staffs are aware of their special needs.

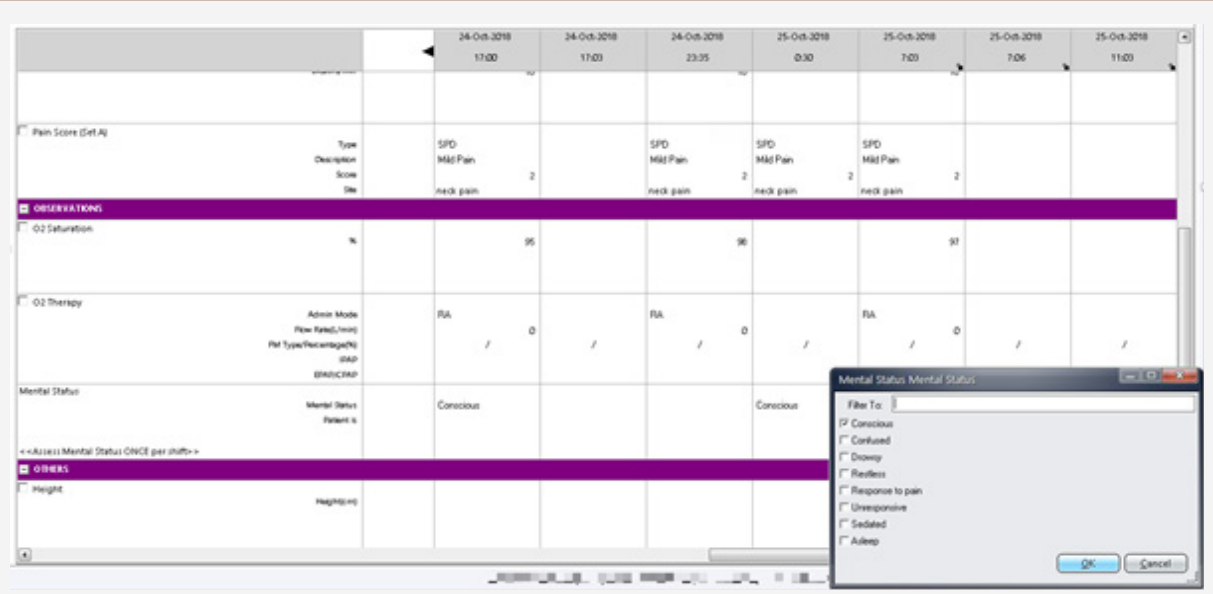

Figure 2: Mental status examination.

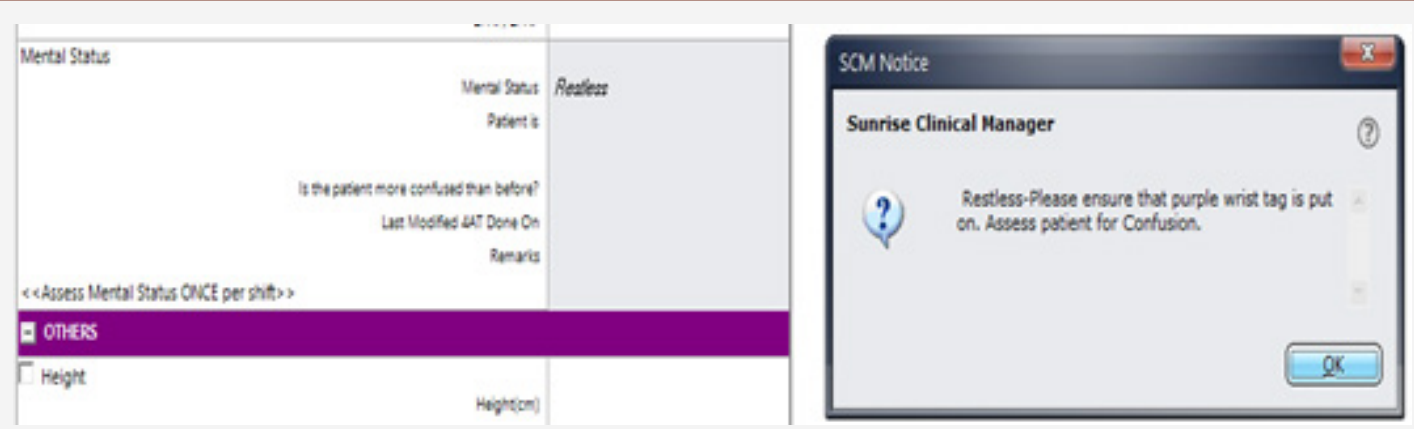

Figure 3: Prompt to put on a purple wrist tag.

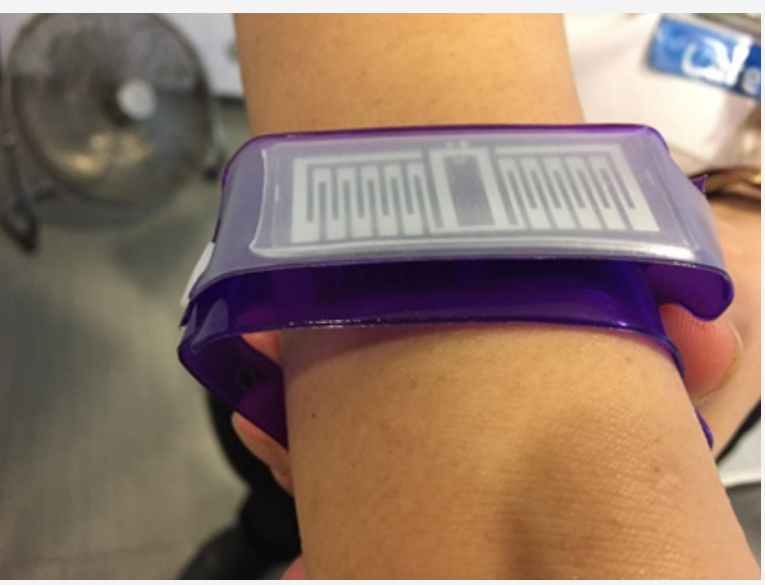

Figure 4: The purple wrist tag which also serves as a RFID device. 


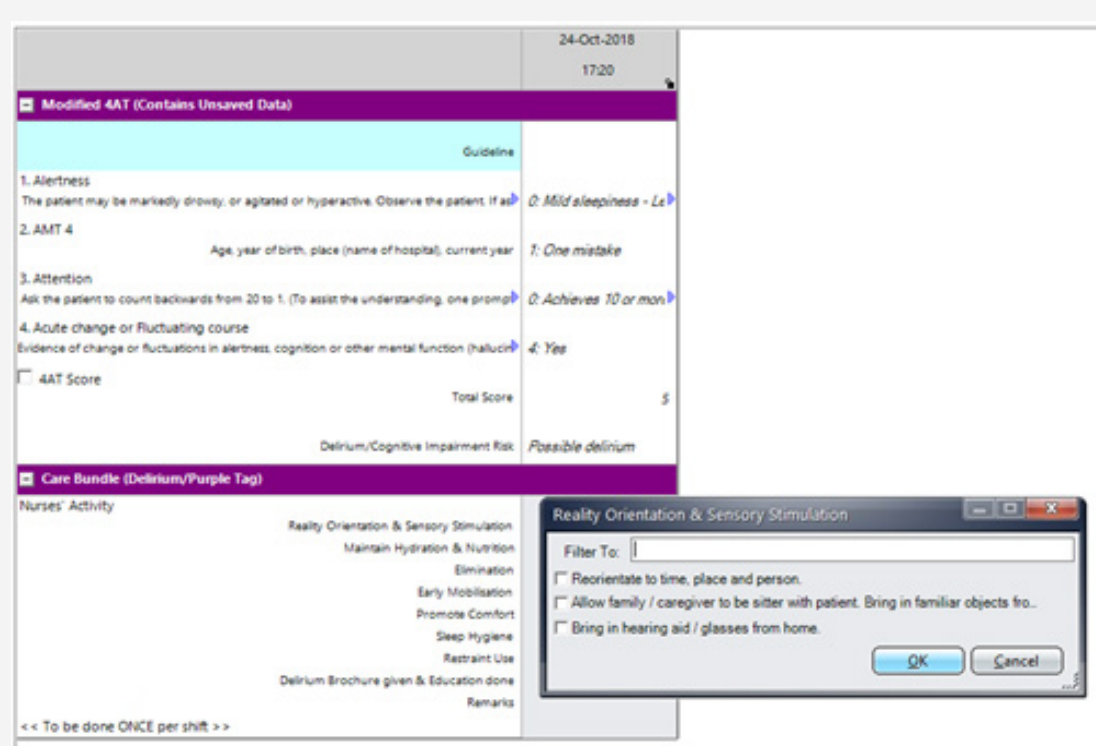

Figure 5: Screening tool for delirium.

Once the change in mental status is noted to be different from the entry from previous shifts, nurses will be directed to the screening tool for delirium, and in the hospital, it is the modified 4AT which is used. (Figure 5) To improve the sensitivity of the 4AT, we have modified the test for attention to counting backwards from 20 to 1 , instead of the original version which is reciting the months of the year backwards. In the local culture, the months of the year is counted numerically as the 1 st month to the 12 th month. Counting backwards 20-1 is validated as check for attention in the local Abbreviated Mental Test [10].

\section{The Purple Wrist Tag}

The purple wrist tag has no diagnostic purpose. The inpatients wearing a purple wrist tag indicate that they have cognitive issues, but the tag is not diagnostic of either delirium or dementia. It does, however, serve another purpose as part of a RFID device. The other part of this hospital wide project involves reducing the rate of cognitively impaired patients absconding from the hospital premises and forget how to return. The argument against restraining patient's freedom of movement was heavily taken into considerations prior to this project. The hospital should not be seen as a high security institution where the patients are confined against their will. The authors are aware of the inappropriateness of restricting patients' autonomy of movement as a basic human right. Therefore, the hospital allows patients' freedom of movement freely within the hospital. However, for their safety purposes, we have to ensure they are accompanied at all times within and outside the hospital's compound. The challenge was therefore to identify patients with cognitive impairment correctly and find a balance between safety and autonomy.

At the exit gantries of the hospital, there are hidden RFID sensors which pick up the signals from the purple wrist tag and an alarm will be triggered should a purple wrist tag pass through the gantries. The security officers will ensure that the patients who wear the purple tags are accompanied before they leave the hospital compound, for their safety. A SMS will be triggered to the nursing officer on duty at the same time the alarm is triggered. For the patients who were not accompanied, they will be discretely escorted back to their respective wards. So far, we have no patients who absconded while wearing the purple wrist tags.

\section{Diagnosis and Management of Delirium}

Once the patients screened positive for delirium on the mental status assessment plus a positive score on 4AT, the junior doctors will be contacted to investigate for delirium causes, including medication review to identify causes of delirium (Figure 1).

Table 1: Common causes of delirium: DELIRIUMSP.

\begin{tabular}{|c|c|}
\hline Drugs & Anticholinergic, analgesia, neuroleptics, sedatives \\
\hline Environment & $\begin{array}{l}\text { Unfamiliar places, unfamiliar persons may } \\
\text { further confuse patients. Ensure spectacles and } \\
\text { hearing aids to be on when patient is in the ward. } \\
\text { Frequent orientation to time, place, person, is also } \\
\text { a way to help patient familiarize himself. }\end{array}$ \\
\hline Low 02 state & $\begin{array}{l}\text { Myocardial infarction which can present silently } \\
\text { in elderly, pulmonary embolism, stroke }\end{array}$ \\
\hline Infection & $\begin{array}{l}\text { Skin, soft tissue, prostate, ear infections are often } \\
\text { missed }\end{array}$ \\
\hline $\begin{array}{l}\text { Retention of urine } \\
\text { or bowel }\end{array}$ & Ensure regular elimination of stool and urine \\
\hline Ictal & $\begin{array}{c}\text { Non convulsive seizure may not be picked up } \\
\text { especially in hypoactive delirium }\end{array}$ \\
\hline $\begin{array}{l}\text { Undernourished } \\
\text { and under } \\
\text { hydration }\end{array}$ & $\begin{array}{l}\text { Elderly often are not meeting their caloric } \\
\text { requirement and can easily became dehydrated }\end{array}$ \\
\hline Metabolites & $\begin{array}{l}\text { Electrolytes abnormalities including magnesium, } \\
\text { phosphate and calcium }\end{array}$ \\
\hline $\begin{array}{c}\text { Subdural } \\
\text { hematoma }\end{array}$ & $\begin{array}{l}\text { For patient with recurrent fall even when he/she } \\
\text { denies head injury }\end{array}$ \\
\hline Pain & Pain from multiple causes \\
\hline
\end{tabular}

The onset of delirium suggests that the elderly has reduced functional reserve, making them more susceptible to external insults. The causes of delirium are often multiple in a single individual, as listed in Table 1 [11]. The hospital has created an order set for working up causes for delirium in the electronic medical record system, which includes simple blood investigations as indicated in Table 1 on the delirium pathway. The other more sophisticated tests, such as computed tomography of the brain, 2D 
Echo, EEG, etc are not included in the routine order set as the yield is low for routine screening. However, the team may request for more sophisticated tests if there are clinical indications for them.

Part of the clinical presentation of delirium is the fluctuations in attention and awareness. The fluctuations may occur on and off throughout the day and the duration is not consistent. Therefore, we suggested for the assessment for mental state to be performed at least 3 times a day to improve the chances of diagnosing delirium early, especially for the elderly who are poorly communicative or the elderly who presents with hypoactive delirium who are lethargic and poorly motivated. The honours of picking up changes and fluctuations in mental status is best observed by the ward nurses, rather than the medical teams since the nurses have longer contact with the patients compared to the team doctors.

\section{Care for the Confused Elderly}

The nurses in both the MB and IB were trained to recognize, diagnose and management of delirium. There are currently delirium champions strategically placed in every ward so they are requested to get themselves updated and keep their junior team members trained. In order to ensure care delivery are equally distributed to all the confused elderly, including cases where the nurses are not aware of the delirum work process or competent in using the $4 \mathrm{AT}$, the team created a care bundle for purple tag on the SCM. The authors are aware of the constant addition of new nurses into the hospital pool of nurses and timely training for every new staff is no mean feat. Besides, there are not many local hospitals where the nurses have received training on caring for the confused elderly.

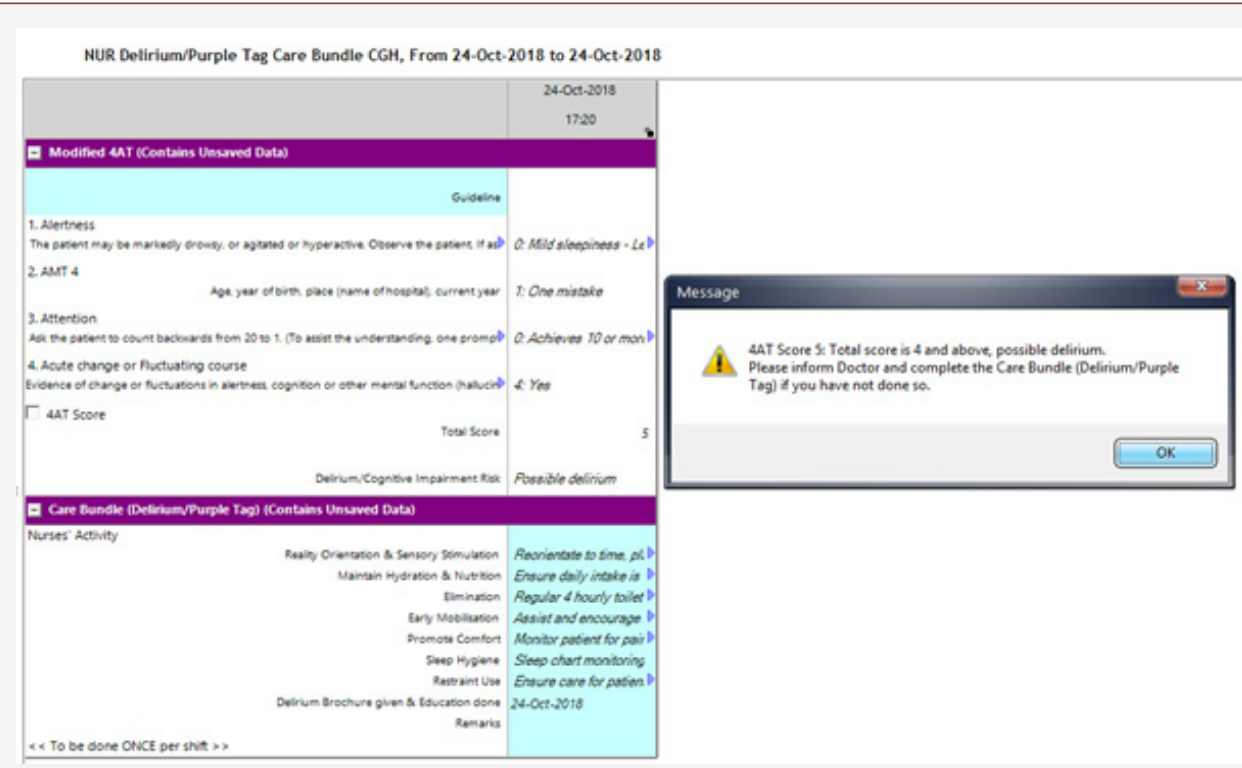

Figure 6: Care bundle for the confused elderly.

The care bundle ensures factors which can potentially contribute to onset of delirium are removed or managed (Table 1 \& Figure 6). These are also the factors which may cause agitation and restlessness among the elderly with dementia as their needs are unmet [12].

\section{Usage of Physical Restraints for the Elderly Inpatients}

The usage of physical restraints as a means of reducing falls is frowned upon in many countries. The usage of restraints is always taught as the last resort when all the other measures failed and the patients are at risk of endangering themselves or others surrounding them. The aim of physical restraints is to enforce immobility so they can be safely nursed in bed. Common indications for restraint usage include reducing fall risk, risks of pulling out medical equipment like catheters or tubes which may be life sustaining or risk of damaging the hospital's properties. Physical restraints have not been shown to reduce fall risk and in fact, it is associated with higher risk of injurious falls. Restraint usage is also against the practice of allowing autonomy of mobility which is a basic human right [13]. The practice of restraint usage in the hospital has dropped since the authors took the initiatives to educate the nursing staff hospital wide on dementia, causes of agitation among the vulnerable elderly and looking at non-pharmacological ways to manage the challenging behaviours. It is important to look beyond the behaviour and search for reversible causes of agitation and restlessness (Table 2) [14], especially among the elderly with dementia who may have difficulties communicating their needs to the care staffs.

Table 2: Causes of agitation among elderly with dementia.

\begin{tabular}{|c|}
\hline Pain- inadequately treated \\
\hline $\begin{array}{c}\text { Restraint use- limits autonomy of movement, both physical and chemical } \\
\text { restraints may worsen agitation }\end{array}$ \\
\hline Delirium- particularly if the change in behavior is a new feature. \\
\hline Physiological needs- hunger, thirst, feeling tired. \\
\hline Social reasons- boredom, loneliness, changes in routines or caregivers. \\
\hline Elimination- Urinary retention, constipation. \\
\hline Drugs- antipsychotics, drugs with anticholinergic side effects. \\
\hline $\begin{array}{c}\text { Environment- new changes, too warm/ cold, an under or overstimulated } \\
\text { environment. }\end{array}$ \\
\hline $\begin{array}{c}\text { Sensory- sensory impairment like visual/ hearing impairment, } \\
\text { misinterpretation of stimuli. }\end{array}$ \\
\hline Emotional needs- anxiety, boredom, feeling frightened, insecure, etc. \\
\hline
\end{tabular}

Currently, the nurses are allowed to initiate an order for restraint usage in the SCM when they are unable to manage the agitated and aggressive patients where all other measures have been tried and 
failed. At initiation, they are prompted to answer if the change in behaviour is new (Figure 7). If the change of behavior is new, it will prompt the nurse to inform team doctor to work up for delirium. Once restraint order is up, it leads to the Restraint protocol (Figure
8). The restraint protocol is put in place to ensure that the patients are not agitated simply because they have unmet needs which have been missed by the nursing or medical staffs, which are potentially reversible.

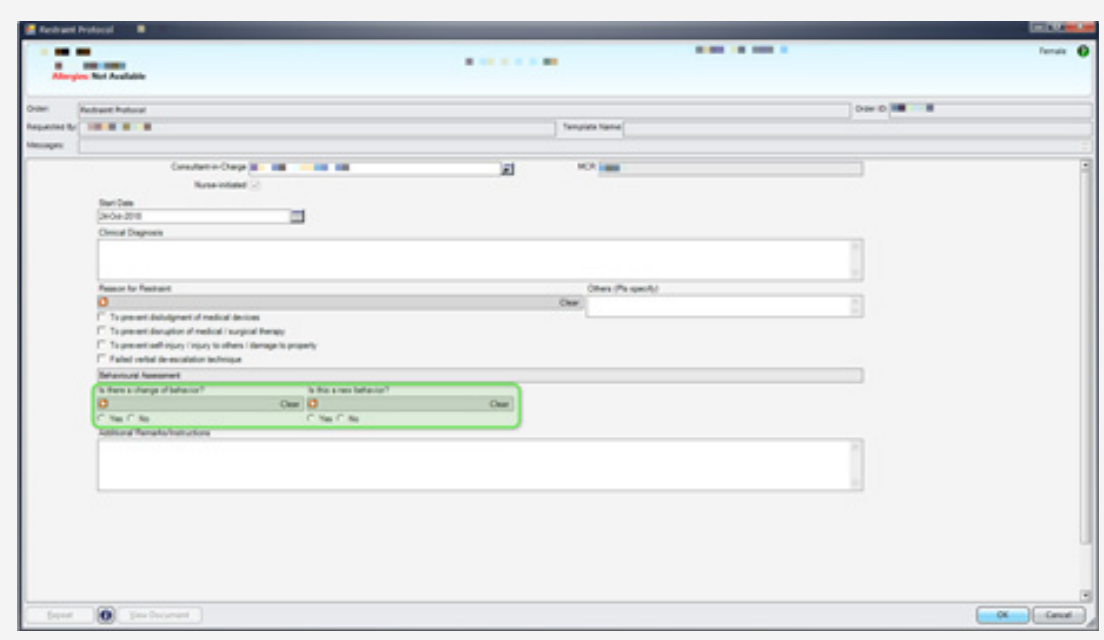

Figure 7: Restraint order.

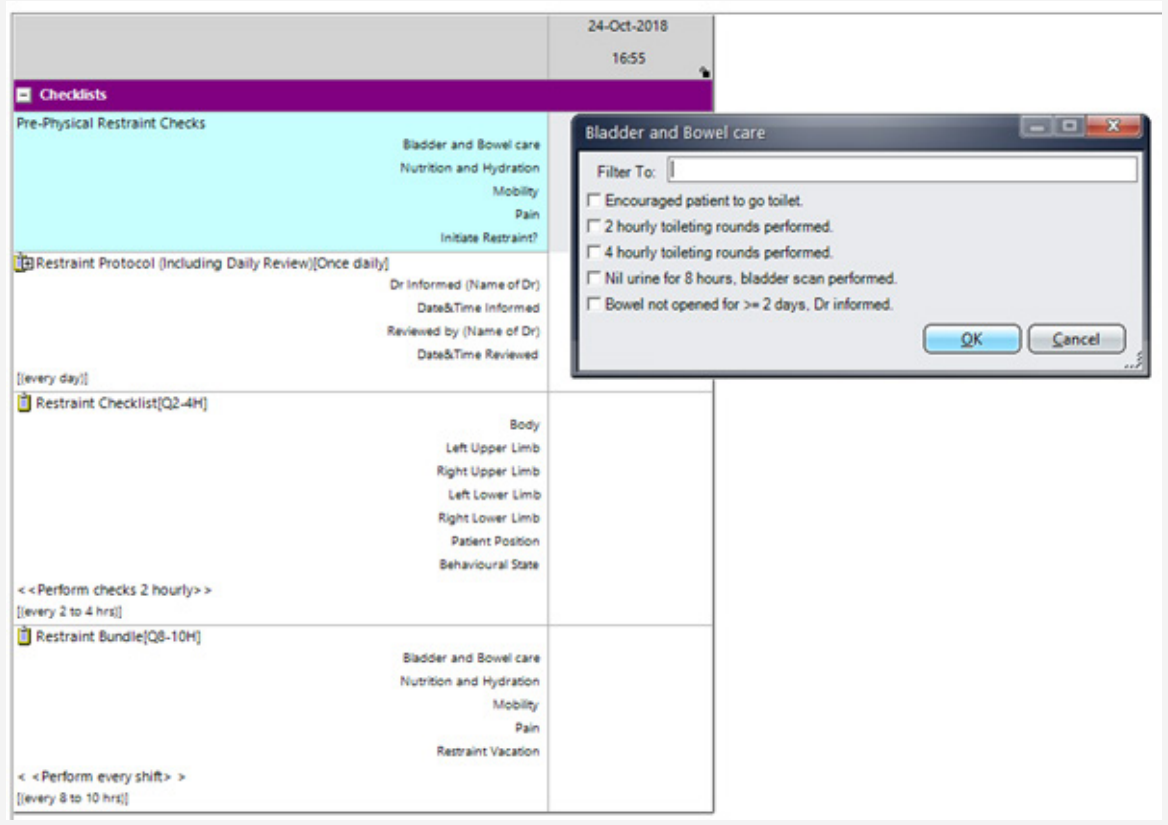

Figure 8: Restraint protocol.

While the patients are being restrained, the nurses are also required to ensure that the patients receive due diligent care and their patients' basic needs are taken care of. There will also be prompts to try off restraints (restraint vacation) once a day, should the patients become calmer. Restraint vacation was felt to be important since the patient's behaviors may fluctuate throughout the day and if their needs are attended to, they may calm down. Besides, prolonged periods of restraint usage cause complications associated with immobility and physical deconditioning.

\section{Discussion}

Delirium is considered as a quality indicator for good health care delivery for the elderly patients [15]. In anticipation of silver tsunami which is fast approaching, there is a need to put in place good care delivery for the elderly patients as geriatricians are scarce resources and it makes sense to train junior doctors and nurses on the ground to understand the unique needs of the elderly patients.

The elderly patients with poor cognitive reserve are more likely to develop incident delirium during their stay in the hospital, which may be preventable. This group of vulnerable elderly may not be able to express themselves clearly to the medical or nursing staff due to their cognitive issues and it is therefore the caregivers' duty to detect changes in them and intervene early before they develop further harm during their stay.

Screening process and work up for delirium are both fast and reliable, but who is the best person to detect a change? The authors felt that the task is better owned by the nursing staff, 
acknowledging that the nurses have another duty to perform in addition to their already tight work schedule. The authors are continuously monitoring for fatigue among the nursing staff, while looking at the hospital data at indicators such as restraint usage, inpatient fall rate and usage of the 4AT. So far, the nurses reported feelings of satisfaction at their improved ability to pick up delirium, understanding the needs of their confused elderly patients and the care bundle acts as reminders while taking care of the elderly patients wearing the purple wrist tags.

\section{Conclusion}

Creating an awareness that changes in mental state among the vulnerable elderly patients in an acute hospital setting may be indicative of delirium was the starting aim of this huge project which is now applicable hospital wide. In order for this huge work flow to progress smoothly among the team, it fosters on good team work, trust and reliance on each other in order to deliver good patient care and reduce complications like falls and restraint usage.

\section{Acknowledgement}

None.

\section{Conflict of Interest}

No conflict of Interest.

\section{References}

1. Department of Statistics Singapore. September 2017 Population trends.

2. Ministry of Health (2017) Hospital admissions by sector, gender and age group.

3. Walsh KA (2007) Review: Hospitalization and the elderly. Ann Long Term Care 15: 18-23.
4. Collins N, Blanchard MR, Tookman A, Sampson EL (2010) Detection of delirium in the acute hospital. Age Ageing 39(1): 131-135.

5. Elie M, Rousseau F, Cole M, Primeau F, McCusker J, et al. (2000) Prevalence and detection of delirium in elderly emergency department patients. CMAJ 163(8): 977-981.

6. John Young, Sharon K Inouye (2014) Delirium in older people. Lancet 383(9920): 911-922.

7. Leslie DL, Marcantonio ER, Zhang Y, Leo-Summers L, Inouye SK (2008) Oneyear health care costs associated with delirium in the elderly population. Arch Intern Med 168(1): 27-32.

8. Inouye SK, van Dyck CH, Alessi CA, Balkin S, Siegal AP, et al. (1990) Clarifying confusion: The Confusion Assessment Method: A new method for detection of delirium. Ann Int Med 113(12): 941-948.

9. Bellelli G, Morandi A, Davis DH, Mazzola P4, Turco R, et al. (2014) Validation of the 4AT, a new instrument for rapid delirium screening: A study in 234 hospitalised older people. Age ageing 43(4): 496-502.

10. Sahadevan S, Lim PP, Tan NJ, Chan SP (2000) Diagnostic performance of two mental status tests in the older Chinese: Influence of education and age cut-off values. Int J Geriatr Psychiatry 15(3): 234-241.

11. Marcantonio ER (2017) Delirium in hospitalized older adults. N Eng J Med 377(15): 1456-1466.

12. SC Lim (2017) Managing behavioural and psychological symptoms of dementia-from a geriatrician's perspective. J Ageing Geriatr Psychiatry $1(3)$.

13. SC Lim (2016) Restraint use in the management of the elderly with dementia in hospital. Internal Med Res Open J 1(2): 1-4.

14. Rosenberg PB, Mielke MM, Appleby BS, Oh ES, Geda YE, et al. (2013) The association of neuropsychatric symptoms in $\mathrm{MCI}$ with incident dementia and Alzheimer disease. Am J geriatr Psychiatry 21(7): 685-695.

15. Shekelle PG, MacLean CH, Morton SC, Wenger NS (2001) Acove quality indicators. Ann Intern Med 135: 635-667. 